\title{
Parámetros cefalométricos para determinar biotipo facial en adultos chilenos.
}

\section{Cephalometric norms related to Facial type in eugnathic Chilean adults.}

\author{
Bárbara Cerda-Peralta ${ }^{1}$, Rolando Schulz-Rosales ${ }^{1 *}$, Jimena López-Garrido ${ }^{1}$, Fernando Romo-Ormazabal ${ }^{1}$
}

1. Clínica Integral del Adulto, Facultad de Odontología, Universidad de Chile, Santiago, Chile.

* Correspondencia autor: Dr. Rolando Schulz Rosales, Profesor Asistente | Facultad de odontología Universidad de Chile | E-mail: rschulz@odontologia.uchile.cl | Teléfono: +562 29781715 | Dirección postal: Sergio Livingstone Pohlhammer No 943 (ex Olivos)

Trabajo recibido el 22/08/2016.

Aprobado para su publicación el 12/10/2016.

\begin{abstract}
RESUMEN
Objetivo: Determinar normas cefalométricas relacionadas con el biotipo facial en una población eugnácica chilena adulta y compararla con los parámetros cefalométricos clásicos. Material y método: Estudio transversal. Se analizaron 96 telerradiografías digitales de adultos jóvenes chilenos eugnácicos entre 18 y 35 años. El análisis cefalométrico fue realizado con el software Quick Ceph $2000 \circledast$. Resultados: El ángulo goníaco tuvo un valor promedio de $121.96^{\circ}$, el ratio de Bjork-Jarabak $67.11 \%$, el ángulo SN-GoGn $32.65^{\circ}$, el ángulo interbasal de Schwarz $23.8^{\circ}$, el VERT de Ricketts un valor de 2.33 y la altura facial inferior de Ricketts un valor de $44.1^{\circ}$. Se observaron diferencias significativas entre hombres y mujeres para el ratio de Bjork-Jarabak, ángulo SN-GoGn, ángulo interbasal de Schwarz y el VERT de Ricketts. Conclusiones: Los parámetros estudiados: ángulo goníaco, ángulo SN-GoGn, ángulo interbasal, altura facial inferior de Ricketts, son similares a las normas cefalométricas establecidas por sus autores originales, a excepción del ratio de Björk-Jarabak y el VERT de Ricketts. Asimismo, existe un fenómeno de dimorfismo sexual significativo, que debe ser tomado en cuenta al momento de diagnosticar a los individuos adultos.

PALABRAS CLAVE:

Biotipo Facial; Cefalometría; Maloclusión; Ortodoncia.
\end{abstract}

Rev. Clin. Periodoncia Implantol. Rehabil. Oral Vol. 12(1); 08-11, 2019.

\section{ABSTRACT}

Objective: The aim of this study was to determine cephalometric standards related to facial type in a eugnathic adult Chilean population and to compare them with the classical cephalometric parameters. Methods: Cross-sectional study. Ninety six eugnathic young Chilean adults (between 18 and 35 years old) were included in this study. Lateral digital standardized cephalometric radiographs were used. The cephalometric analysis used Quick-Ceph $2000{ }^{\circledR}$ software. Results: The mean value of the gonial angle was $121.96^{\circ}$, the Biörk-Jarabak ratio was $67.11 \%$, SN-GoGn angle was $32.65^{\circ}$, interbasal angle (Schwarz) was $23.8^{\circ}$, Ricketts VERT was 2.33 and Ricketts lower facial height $44.1^{\circ}$. There was a significant difference between men and women for the Biörk-Jarabak ratio, SN-GoGn, interbasal angle and Ricketts VERT. Conclusion: Cephalometric standards obtained are similar to those established by the original authors, except for the BjörkJarabak ratio and Ricketts lower facial height. However, there is a significative sexual dimorphism that should be considered when making a patient's diagnosis..

KEY WORDS:

Facial type; Cephalometrics; Malocclusion; Orthodontics.

Rev. Clin. Periodoncia Implantol. Rehabil. Oral Vol. 12(1); 08-11, 2019.

\section{INTRODUCCIÓN}

El biotipo facial corresponde a un conjunto de características morfodiferenciales de individuos de la misma especie, en relación a ciertos rasgos en común del esqueleto facial, determinados por la genética y el ambiente ${ }^{(1)}$. La utilidad del biotipo facial es transversal en ortodoncia, cirugía máxilo facial y rehabilitación oral.

En cuanto a la rehabilitación oral de los pacientes desdentados totales y parciales, sus objetivos fundamentales son: conservar el remanente biológico, restablecer la función y devolver la estética perdida. Los cambios que sufre el paciente a lo largo de su vida se deben no sólo al desdentamiento, sino también a modificaciones esqueletales derivadas de la reabsorción del hueso alveolar, migración dentaria patológica, alteración del plano oclusal y remodelación de las ATMs.

Para la restitución de las estructuras dentarias y las dimensiones faciales, el análisis de la dimensión vertical es fundamental. Ésta tiene una íntima relación con el biotipo facial. Por ello, la determinación del biotipo tanto clínica como cefalométrica presenta un desafío en pacientes 
desdentados totales o parciales con alteraciones en la dimensión vertical, pues no se describen parámetros para estos individuos. Además, en ellos puede ser necesario también ajustar la técnica de toma radiográfica ${ }^{(2)}$

En cuanto a la clasificación de biotipo, de acuerdo a las proporciones del esqueleto facial se encuentran:

- Dolicofacial: Predomina el largo de la cara por sobre ancho.

- Braquifacial: El ancho facial es predominante por sobre el alto.

- Mesofacial: Existe un equilibrio entre ambas dimensiones.

\section{Características de los biotipos faciales ${ }^{(3)}$ :}

Dolicofacial: Posee mandíbulas con ramas poco desarrolladas en relación al cuerpo, cara alargada, arcadas dentarias angostas. Presentan divergencia entre la mandíbula y la base craneal y entre la base mandibular y el plano biespinal. Predominando el largo sobre el ancho en las dimensiones esqueletales. Su dirección de crecimiento es vertical (Fig 1a).

Mesofacial: Su dirección de crecimiento es normal, con sus diámetros vertical y transversal proporcionados, los maxilares y arcadas dentarias son de configuración similar, el crecimiento va en dirección hacia abajo y hacia adelante.

Braquifacial: Presentan mandíbulas con ramas potentes, caras anchas, arcadas dentarias bien desarrolladas, existe un mayor desarrollo muscular, dirección de crecimiento horizontal; éste se manifiesta por una rotación anterior de la sínfisis mandibular y el eje facial tiende a girar hacia adelante y arriba. El mentón es prominente y el surco mentolabial usualmente es marcado (Fig 1b).

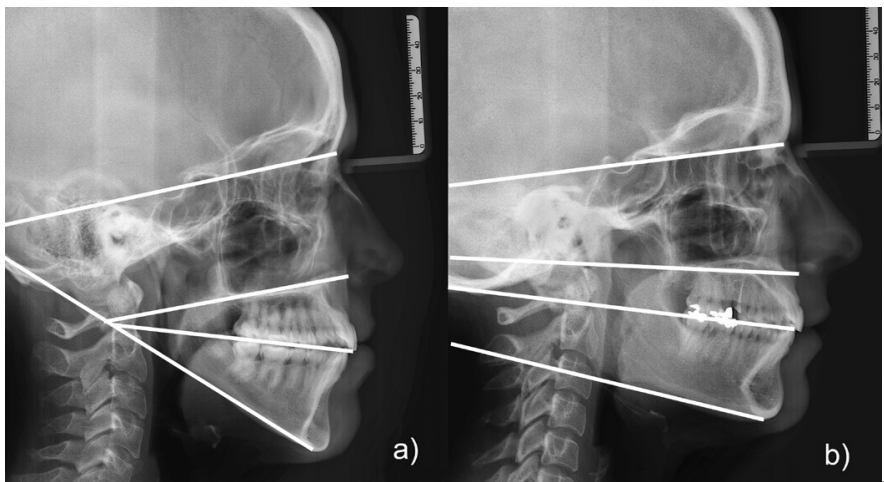

Figura 1. Telerradiografías correspondientes a individuos dólicofacial (a) y braquifacial (b). Nótese la forma de la mandíbula y la divergencia de los planos faciales

La cefalometría corresponde a un protocolo de mediciones precisas sobre la telerradiografía, que permite objetivar las relaciones entre las estructuras óseas y dentarias, mediante la medición de ángulos, distancias y proporciones. Las medidas obtenidas se comparan con "Normas" establecidas a través de estudios poblacionales representativos ${ }^{(4)}$. Las normas cefalométricas que habitualmente se utilizan son obtenidas de niños en crecimiento y/o poblaciones diferentes a la chilena por lo tanto el patrón de referencia podría no ser adecuado para estos individuos ${ }^{(5)}$. A raíz de esto, para personas adultas es necesario contar con referencias cefalométricas que sean un punto de partida para planificar un tratamiento odontológico rehabilitador.

Referencias cefalométricas relacionadas con el Biotipo Facial:

Las referencias utilizadas para evaluar Biotipo Facial atribuyen importancia a la dirección de crecimiento facial, a aspectos cualitativos y cuantitativos del crecimiento cráneo mandibular y al desarrollo mandibular propiamente tal. Estas referencias corresponden a:

Ángulo mandibular o ángulo goníaco: Este parámetro es independiente de la edad y el tiempo de desdentamiento e indica la relación entre el cuerpo y la rama mandibular. Se traza la tangente al borde posterior de la mandíbula y la tangente al borde inferior mandibular. La norma de este ángulo es $123^{\circ} \pm 10^{\circ}$. Un ángulo aumentado da señal de un menor desarrollo de la rama mandibular en relación al cuerpo, característico de los pacientes dolicofaciales y por el contrario un ángulo disminuido es típico de los individuos braquifaciales, en los cuales se encuentra una equivalencia entre el cuerpo y la rama mandibular ${ }^{(6,7)}$ (Fig 2)

Análisis de Bjork-Jarabak: Los biotipos faciales fueron definidos en el análisis de Bjork-Jarabak en función de la relación proporcional (ratio) de las alturas faciales posterior y anterior, relación que da cuenta del grado
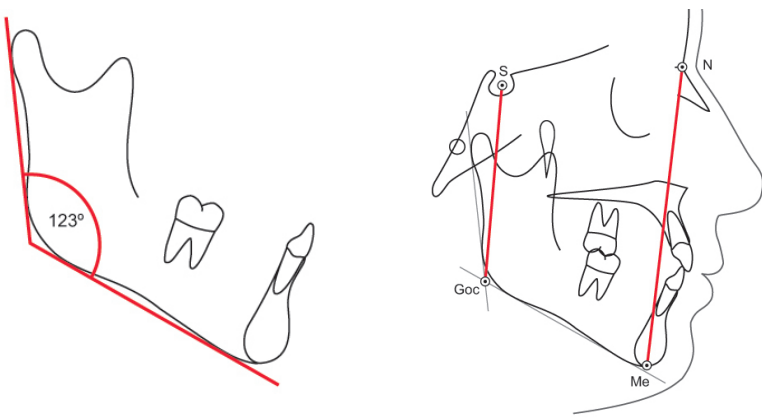

Figura 2. a. Ángulo Goníaco o ángulo mandibular. b. Análisis de BjorkJarabak a través de la altura facial anterior $(\mathrm{N}-\mathrm{Me})$ y altura facial posterior (S-Go).

de divergencia del plano mandibular respecto del plano silla-nasion ${ }^{(8)}$ (Fig. 3). Se obtiene con la fórmula:

Biotipo Facial $=($ Altura Facial Posterior/Altura Facial Anterior $) * 100$.

Hiperdivergente (Dólicofacial), si el resultado obtenido está entre $54 \%$ y $58 \%$. La cara será de tipo retrognático, con una dirección de crecimiento en el sentido de las manecillas del reloj, también denominado crecimiento rotacional posterior.

Normodivergente (Mesofacial), si el resultado obtenido va entre el 59 al $63 \%$. Corresponde a un crecimiento neutral, con una dirección de crecimiento directamente hacia abajo, sin rotación en ninguno de los dos sentidos.

Hipodivergente (Braquifacial), si el resultado obtenido va entre el 64 y $80 \%$. Presenta una dirección de crecimiento en sentido contrario a las manecillas del reloj, también denominado crecimiento rotacional anterior ${ }^{(8)}$.

Divergencia facial de Downs-Steiner: Steiner: Ángulo Silla-Nasion Gonion-Gnation, define el tipo de crecimiento mandibular, mide la convergencia o divergencia de la mandíbula en relación a la base cranea anterior. Para determinarlo se traza el plano Silla-Nasion y el Plano Mandibular Presentando una norma de $32^{\circ}+2$ según Steiner y de $32^{\circ} \pm 5$ según Riedel (Fig. 4). Un ángulo mayor a la norma indica que estamos frente a un paciente dolicofacial. Por otra parte, un ángulo menor indica

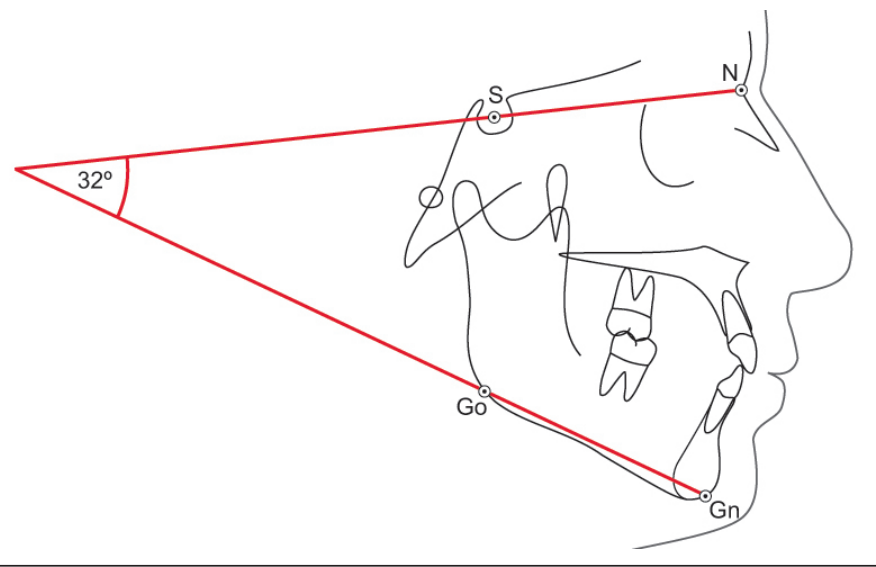

Figura 3. Ángulo Silla Gonion-Gnation

que el individuo tiene la cara más bien acortada(9)

Altura facial inferior de Ricketts: Es equivalente al ángulo B de Schwarz (Fig. 5). Corresponde al ángulo formado entre la línea Xi-ENA y Xi- Pm. Un ángulo mayor que la norma indica divergencia entre la mandíbula y el maxilar superior, correspondiendo a un patrón dolicofacial con musculatura débil y tendencia a mordida abierta esqueletal. De manera inversa valores menores que la norma indican patrones braquifaciales, con musculatura fuerte y generalmente con mordida profunda ${ }^{(2)}$.

Ángulo B de Schwarz: Representa la actividad masticatoria y en consecuencia el desarrollo muscular. Este ángulo tiene un valor de $20^{\circ}$ $+5^{\circ}$. Para determinarlo se traza el plano Biespinal (ENA-ENP) y la Línea mandibular de Schwarz (Punto Mentón hasta la parte más profunda de la escotadura maseterina). Un ángulo mayor a la norma representa a un individuo dolicofacial(3) y podría también presentarse mordida abierta anterior (Fig 4b). 


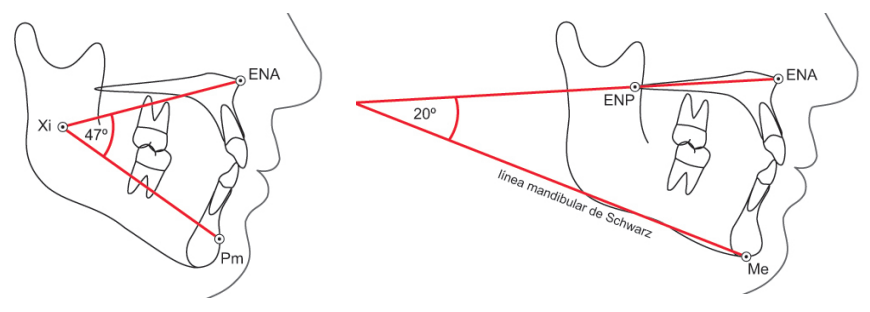

Figura 4. a. Altura Facial Inferior (Ricketts). b. Ángulo B de Schwarz.

Índice VERT de Ricketts: Define el biotipo facial del paciente, corresponde a un coeficiente de variación que establece numéricamente el tipo y la cantidad de crecimiento vertical del tercio inferior del rostro, provocado por la rotación posterior o anterior de la mandíbula. Incluye 5 ángulos que relacionan la posición mandibular, estos son: eje facial, profundidad facial, plano mandibular, altura facial inferior, y arco mandibular ${ }^{(1)}$. Se obtiene con la siguiente fórmula:

VERT $=(($ Eje Facial -90$) / 3)+(($ Profundidad facial-90)/3 $)+((24.5-$ Plano Mandibular $) / 4)+((47-$ Altura Facial Inferior $) / 4)+(($ Arco mandibular $-28.5) / 5)$

El propósito de este estudio fue determinar normas cefalométricas relacionadas con el biotipo facial en una población eugnácica chilena adulta, permitiendo obtener referencias aplicables en Chile. Para este estudio se analizaron adultos jóvenes con buena oclusión que no hubieran sufrido ningún deterioro de su sistema estomatognático, concepto conocido como eugnacia ${ }^{(10)}$

\section{MATERIAL Y MÉTODO}

Estudio transversal. Se analizaron 96 telerradiografías digitales tomadas el año 2011 de adultos jóvenes chilenos eugnácicos entre 18 y 35 años, pertenecientes a un base de datos de un estudio previo, de la Clínica Integral del Adulto de la Facultad de Odontología, Universidad de Chile.

\section{Criterios de inclusión y exclusión}

Se incluyeron en este estudio individuos con dentición natural completa, clase esqueletal I o II leve (sin necesidad de tratamiento), sin apiñamiento dentario (IOTN menor o igual a 2)(19), presencia de acople anterior y ATM con rangos de movilidad normales, sin síntomas asociados. Fueron excluidos de este estudio sujetos con cualquier enfermedad que haya provocado una alteración del crecimiento vertical, pacientes en tratamiento de ortodoncia previo o en curso, pacientes sometidos previamente a cirugía ortognática u otra cirugía que altere la morfología facial, malos hábitos orales, apiñamiento dentario moderado o severo.

\section{Análisis cefalométrico y estadístico}

Se realizó un análisis cefalométrico con el software Quick Ceph $2000^{\circledR}$ versión 3.4 (Quick Ceph Systems $^{\circledR}$ ), utilizando las referencias relacionadas con biotipo facial.
El análisis estadístico se realizó con el software Stata ${ }^{\circledR}$ (Stata Corporation L.P).

\section{RESULTADOS}

La muestra incluyó 48 hombres y 48 mujeres. El ángulo goníaco tuvo un valor promedio de $121.96^{\circ} \pm 6$, el ángulo altura facial inferior tuvo un valor promedio de $44.1^{\circ}$, sin diferencia significativa entre sexo para estos dos parámetros.

El ratio de Bjork-Jarabak arrojó un valor de $67.11 \% \pm 5.15$, el ángulo SN-GoGn $32.65^{\circ} \pm 6.15$, el ángulo interbasal $23.8^{\circ} \pm 5.57$ y el VERT de Ricketts un valor de $2.33 \pm 5.14$. Se observaron diferencias significativas entre hombres y mujeres para estos parámetros (Tabla 1).

\section{DISCUSIÓN}

Una definición fundamental al determinar normas cefalométricas para una población, es establecer si se utilizará el criterio de oclusión normal o un perfil facial equilibrado. En esta investigación se propuso encontrar promedios basados en una población eugnácica; es decir, se privilegió el criterio de oclusión normal.

Los resultados obtenidos evidencian valores bastante cercanos a la norma clásica en el ángulo goníaco, SN-GoGn, ángulo B de Schwarz y altura facial inferior de Ricketts ${ }^{(2,3)}$. Sin embargo, el Ratio de Bjork-Jarabak y el VERT están fuertemente desplazados hacia el biotipo braquifacial. Lo anterior muestra que no existe concordancia entre el biotipo determinado por cada uno de los cuatro parámetros por separado, ocurriendo un traslapamiento de biotipos dolicofacial suave o braquifacial suave $\mathrm{e}^{(11,12)}$ Esto incide en las decisiones clínicas, pues dependiendo del método escogido será el biotipo encontrado en el paciente.

Por otra parte, se evidencia un dimorfismo sexual en casi todos los parámetros, exceptuando el ángulo goníaco y altura facial inferior. En el caso de SN-GoGn y ángulo B de Schwarz el ángulo medido es menor en hombres, mientras que el Ratio de Bjork-Jarabak y el VERT es menor en mujeres, siendo estas diferencias significativas. En la literatura se describen diferencias significativas en mandíbulas de hombres y mujeres. Recientemente Alarcón J.A. y cols. (2015) realizaron un estudio analizando el dimorfismo sexual en mandíbulas, comparando tres biotipos faciales. Para determinar este último utilizaron el ángulo entre el plano de Frankfurt y el plano mandibular. Los resultados obtenidos concuerdan con los presentados en este estudio, existiendo diferencias significativas en el análisis por sexo de cada biotipo ${ }^{(13)}$. Todo anterior se describe también en estudios en distintas poblaciones ${ }^{(14,15)}$.

El predominio braquifacial en este estudio se explica por los criterios de inclusión y exclusión, pues al requerirse características eugnácicas se privilegian rasgos tales como la simetría, dientes alineados y musculatura bien desarrollada, asociados con características esqueletales braquifaciales ${ }^{(16,17)}$. Al contrario, las características excluidas tales como el apiñamiento, mordida cruzada, asimetrías faciales, deglución atípica e incompetencia labial, se asocian a características esqueletales dólicofaciales ${ }^{(18)}$

En el VERT, los valores obtenidos para población chilena discrepan

Tabla 1. Promedios de angulación en cada parámetro medido para la muestra en general, hombres y mujeres, comparado con la norma según bibliografía.

\begin{tabular}{|c|c|c|c|c|}
\hline Parámetro & $\begin{array}{c}\text { Norma Según } \\
\text { bibliografía }\end{array}$ & Muestra total $n=96$ & Hombres $n=48$ & Mujeres $n=48$ \\
\hline Angulo goníaco & $123^{\circ} \pm 10^{\circ}$ & $\begin{array}{l}121.96^{\circ} \\
s d=6.17\end{array}$ & $\begin{array}{l}120.99^{\circ} \\
\mathrm{sd}=6.51\end{array}$ & $\begin{array}{l}122.94^{\circ} \\
s d=5.70\end{array}$ \\
\hline *Ratio Bjork-Jarabak (\%) & $59 \%$ a $63 \%$ & $\begin{array}{l}67.11 \% \\
s d=5.15\end{array}$ & $\begin{array}{l}69.00 \% \\
s d=6.13\end{array}$ & $\begin{array}{l}65.21 \% \\
\mathrm{sd}=4.47\end{array}$ \\
\hline *SN-GoGn & $32^{\circ} \pm 5$ & $\begin{array}{c}32.65^{\circ} \\
s d=6.15\end{array}$ & $\begin{array}{c}30.69^{\circ} \\
s d=6.12\end{array}$ & $\begin{array}{c}34.61^{\circ} \\
\mathrm{sd}=5.59\end{array}$ \\
\hline *Interbasal B Schwarz & $20^{\circ} \pm 5^{\circ}$ & $\begin{array}{c}23.8^{\circ} \\
\mathrm{sd}=5.57\end{array}$ & $\begin{array}{c}22.64^{\circ} \\
\mathrm{sd}=5.55\end{array}$ & $\begin{array}{c}24.95 \\
s d=5.41\end{array}$ \\
\hline *VERT (martins \& vigorito) & 0 & $\begin{array}{c}2.33 \\
s d=5.14\end{array}$ & $\begin{array}{c}3.48 \\
\mathrm{sd}=5.25\end{array}$ & $\begin{array}{c}1.17 \\
s d=4.8\end{array}$ \\
\hline Altura facial inferior (Ricketts) & $47^{\circ} \pm 4$ & $\begin{array}{c}44.1^{\circ} \\
s d=3.87\end{array}$ & $\begin{array}{c}43.62^{\circ} \\
s d=3.99\end{array}$ & $\begin{array}{c}44.56^{\circ} \\
s d=3.73\end{array}$ \\
\hline
\end{tabular}

* diferencia significativa entre mujeres y hombres (Ttest) $p<0.05$ 
de manera significativa respecto la norma descrita por Ricketts. Lo que sugiere que este parámetro podría ser impreciso al determinar el biotipo facial, puesto que la norma presenta un valor absoluto que no se reproduce en una población chilena.

En los ángulos Goníaco y SN-GoGN, se encontraron los mismos valores promedio de los autores originales, sólo sería necesario ajustar los rangos de variación para la población chilena adulta. Así, el ángulo goníaco quedaría en $122^{\circ} \pm 6$ y el SN-GoGn en $32^{\circ} \pm 6$.

Finalmente, es interesante notar que el ángulo SN-GoGN con el valor de variación ajustado a la población chilena adulta, podría ser utilizado como único parámetro de determinación del biotipo facial debido a su sencillez y alto grado de confiabilidad.

\section{CONCLUSIONES}

Los parámetros estudiados son similares a las normas cefalométricas establecidas por sus autores originales, a excepción del ratio de Björk-Jarabak y el VERT de Ricketts. Asimismo, existe un fenómeno de dimorfismo sexual significativo, que debe ser tomado en cuenta a momento de diagnosticar a los individuos.

Por otra parte, los métodos utilizados muestran que no existe concordancia entre ellos al determinar biotipo facial. Por ello se recomienda utilizar en conjunto el ángulo goníaco, SN-GoGn, ángulo $B$ de Schwarz y altura facial inferior de Ricketts pues permiten ser más precisos en población chilena. Además, para el ángulo goníaco y SnGoGn se proponen rangos más amplios que los propuestos por los autores, adaptándolos a la población chilena, siendo $122^{\circ} \pm 6$ y $32^{\circ} \pm 6$ respectivamente.

\section{CONFLICTOS DE INTERÉS}

Los autores del trabajo declaran no tener conflicto de intereses.

\section{RESPONSABILIDADES ÉTICAS}

\section{Protección de personas y animales.}

Los autores declaran que para esta investigación no se han realizado experimentos en seres humanos ni en animales.

\section{Confidencialidad de los datos}

Los autores declaran que han seguido los protocolos de su centro de trabajo sobre la publicación de datos de pacientes.

\section{Derecho a la privacidad y consentimiento informado.}

Los autores han obtenido el consentimiento informado de los pacientes y/o sujetos referidos en el artículo. Este documento obra en poder del autor de correspondencia.

\section{Bibliografía}

1. Martins L, Vigorito J. Photometric Analysis Applied in Determining Facial Type. Dental Press J Orthod. 2012 Oct; 17(5): 71-75.

2. Schulz R, Romo F, Cerda B, Moya M. Visualización del plano oclusal y guía anterior en desdentados parciales en la telerradiografía lateral: Descripción de la técnica a través de casos clínicos. Int J Odontostomat. 2013; 7(3):471- 476.

3. Gregoret J. Instrumentación de la cefalometría estática lateral. En: Ortodoncia y cirugía ortognática, diagnóstico y planificación. Barcelona. Ed. Espaxs S.A 1997. p. 227

4. Zamora C. Compendio de cefalometría. $2^{\circ}$ ed. Colombia. Ed. Amolca; 2010. P. 8-16.

5. Montt J, Miquel M, Oyonarte R. Características cefalométricas en jóvenes con oclusión normal y perfil armónico en población chilena. Int J Morphol. 2015; 33(1): 237-244.

6. Siriwat $P$, Jarabak J. Malocclusion and Facial Morphology; is there a relationship? Angle Orthod. 1985; 55(2):127-38.

7. Ildiko M, Ákos P, Irinel P. Radiographic Comparison of vertical skeletal and dental parameters in skeletal open bite. Acta Med Marisiensis. 2016; 62(3): 330-333.

8. Barahona J., Benavides J. Principales análisis cefalométricos utilizados para el diagnóstico ortodóntico. Rev Cient Odontol. 2006; 2(1): 11- 27.

9. Steiner, C.C.: Cephalometrics in clinical practice. Angle Orthod.1959; 29: 8-29. Riedel RA. The relation of maxillary structures to cranium in malocclusion and in normal occlusion. Angle Orthodontist 1952; 22(3):142-145
10. Gianni, E. La Nueva Ortognatodoncia. Italia, Ed. Piccin, 1989. p. 3

11. Claro C; Abrao J, Reis S. Association between overbite and craniofacial growth pattern. Braz. oral res. 2010, 24 (4): 425-32.

12. Benedicto Ede N, Kairalla SA, Oliveira GM, Junior LR, Rosário HD, Paranhos LR. Determination of vertical characteristics with different cephalometric measurements. Eur J Dent. 2016 Jan-Mar; 10(1):116-20.

13. Alarcón, J.A., et al. Variation of mandibular sexual dimorphism across human facial patterns. HOMO - J. Comp. Hum. Biol. In Press 2016

14. Upadhyay RB, Upadhyay J, Agrawal P, Rao NN. Analysis of gonial angle in relation to age, gender, and dentition status by radiological and anthropometric methods. J Forensic Dent Sci. 2012; 4(1): 29-33.

15. Rodriguez-Cardenas YA, Arriola-Guillen LE, Flores-Mir C. Björk-Jarabak cephalometric analysis on CBCT synthesized cephalograms with different dentofacial sagittal skeletal patterns. J Orthod. 2014; 19(6): 46-53.

16. Kiliaridis, S, Kälebo, P. Masseter muscle thickness measured by ultrasonography and its relation to facial morphology. J Dent Res. 1991; 70(9): 1262-5.

17. Satiroğlu $F$, Arun $T$, lik F. Comparative data on facial morphology and muscle thickness using ultrasonography. Eur J Orthodont. 2005; 27(6): 562-7.

18. Doyne B. Treatment of a patient with a mutilated Class II, Division 1 malocclusion and a dolichofacial skeletal pattern. Am J Orthod Dentofac Orthop 1995; 108: 542-6. 19. Johansson AM, Follin ME. Evaluation of the dental health component of the index of orthodontic treatment need, by Swedish orthodontists. Eur J Orthod. 2009;31:184-8. 